\title{
Detection of neuropathy using a sudomotor test in type 2 diabetes
}

\author{
Pratiksha G Gandhi' \\ Gundu HR Rao \\ 'IPC Heart Centre, Mumbai, India; \\ ${ }^{2}$ University of Minnesota, Minneapolis, \\ MN, USA
}

Correspondence: Pratiksha G Gandhi A 103/I04, Prathamesh Building, Raghuvanshi Mill Compound, Senapati Bapat Marg, Lower Parel (W),

Mumbai - 400013 , India

Tel +9| 9022530033

Fax +l 9094509625

Email chairpersonipc@yahoo.co.in
This article was published in the following Dove Press journal:

Degenerative Neurological and Neuromuscular Disease

9 January 2015

Number of times this article has been viewed

Background: The sudomotor test is used to evaluate the postganglionic cholinergic sympathetic nervous system. The aim of this study was to evaluate the efficacy of a sudomotor testing device to detect peripheral distal neuropathy (PDN) and cardiac autonomic neuropathy (CAN) in patients with type 2 diabetes.

Materials and methods: A total of 133 type 2 diabetic patients were included in the study. The patients underwent examination at the IPC Heart Care Centre (Mumbai, India) in order to assess the diabetic neuropathy symptoms (DNS) score, using a questionnaire, and the CAN score, using heart rate variability analysis and Ewing tests. In addition, patients were given a sudomotor test using the SudoPath ${ }^{\mathrm{TM}}$ system. The diagnosis of PDN is based on the DNS score. A DNS score of 1 or higher is defined as a positive result for PDN. According to the DNS score, the patients were separated into two groups: Group 1 comprised 35 patients ( 21 males), with the mean age of 66 years (standard deviation $[\mathrm{SD}]=12.1$ ), who had a DNS score $\geq 1$. Group 2 comprised 98 patients (65 males), with the mean age of 56 years ( $\mathrm{SD}=9.6$ ), who had a DNS score $=0$. The SudoPath system is a galvanic skin response device that uses the quantitative sudomotor axon reflex approach to assess for small and unmyelinated fiber neuropathy. The system provides a sudomotor response (SMR) score based on these three measured sudomotor parameters. A statistical analysis was performed using the analysis of variance to compare mean differences between the groups as well as receiver operating characteristic (ROC) curves, to determine the specificity and sensitivity of SMR score to detect PDN, comparing the diabetic groups 1 and 2, and the coefficient of correlation between the CAN score and the SMR score in all the subjects included in the study.

Results: When comparing the diabetes groups 1 and 2, the SMR Score had a sensitivity of $91.4 \%$ and specificity of $79.1 \%$ (cutoff number $>3$ ) to detect PDN ( $P=0.0001)$. Area under the ROC curve $(A U C)=0.893$. A correlation analysis of the CAN score and SMR score returned a coefficient of correlation $r=0.68(P<0.0001)$.

Conclusion: The SudoPath system is easy to use, operator-independent, and fast (3-minute testing time). This study shows that the device will be useful to assess the susceptibility of type 2 diabetes patients in developing PDN complications.

Keywords: peripheral distal neuropathy, cardiovascular autonomic neuropathy, SudoPath system, diabetes treatment management

\section{Introduction}

Sudomotor function (or sweat gland activity) is controlled by a division of the sympathetic system, the postsympathetic cholinergic nerve fibers, which release acetylcholine. The acetylcholine increases microvascular skin flow and provokes sweat output. ${ }^{1,2}$ The 
microcirculatory vessels (capillaries) mediate vasodilation (hyperemia), increased skin temperature, and sweat output. $^{3}$

Sudomotor dysfunction is defined as decreased sudomotor activity. Similar to endothelial dysfunction in the prediction of atherosclerosis, the pathological state known as sudomotor dysfunction is the earliest clinically detectable stage of autonomic neuropathy. ${ }^{4}$ Sudomotor function is affected by the reduced C-fibers density of active sweat glands and/or microcirculatory disorders, which may be associated with chronic pain and/or tingling in the toes. ${ }^{5}$ The main cause of sudomotor dysfunction is diabetes. However, it has been found in different diseases (ie, vitamin deficiency, Parkinson's disease, HIV/AIDS, amyotrophic lateral sclerosis, hypothyroidism, kidney and liver diseases, alcoholism, Alzheimer's disease, and Guillain-Barré syndrome) and/or medication side effects, such as in cancer treatment, antihypertensive treatment (in particular, beta and alpha blockers, and calcium antagonists), and metformin treatment.

Peripheral distal neuropathy (PDN) is a common complication of diabetes. The prevalence of PDN is around $22 \%$ in type 2 diabetes according to a cohort study, and it is associated with neuropathic pain or loss of sensation. ${ }^{6,7}$ PDN can lead to neuropathic ulcers, and it is a leading cause of amputation. ${ }^{8-10}$ Different types of peripheral neuropathy have been identified depending on the damage of the type of nerves (ie, motor, sensory, or autonomic). ${ }^{11}$ Therefore, PDN diagnosis is based on various symptoms and is determined by different medical devices and techniques used for testing, including:

1. nerve conduction study to evaluate myelinated large fibers;

2. touch and vibration, to measure the function of large myelinated A-alpha and -beta sensory fibers;

3. thermal stimuli, to evaluate the pathology of small myelinated (A-delta) fibers; and

4. the sudomotor test, a highly sensitive detection tool used in small unmyelinated nerve fibers or small C-fiber neuropathy. ${ }^{1}$

All of these tests are time-consuming, require special equipment and specialist interpretation, and, except in some specialized centers, are not available in daily practice at physician offices. Therefore, a fast diagnostic method to detect PDN has been created, based on the diabetic neuropathy symptom (DNS) score. ${ }^{12}$

A DNS score of 1 or higher indicates the presence of PDN. The DNS score is based on four validated symptoms to detect PDN with high predictive value, in diabetic patients. ${ }^{12}$
Symptoms of 1) unsteadiness in walking, 2) neuropathic pain, 3) paresthesia, and 4) numbness are elicited. The presence of one symptom is scored as 1 point, and a score of 1 or higher is defined as a positive result for PDN.

The prevalence of confirmed cardiovascular autonomic neuropathy (CAN) in diabetics is around 20\%. However, prevalence can increase up to $65 \%$ with age and duration of diabetes. ${ }^{13}$ Institutional bodies use CAN as a risk marker of mortality and cardiovascular morbidity, and have noted CAN could promote diabetic nephropathy. ${ }^{13}$

CAN was also found to be associated with the risk of hypoglycemia during diabetes treatment. ${ }^{14}$ Symptoms of CAN may include dizziness and fainting upon standing, urinary problems, urinary incontinence, sexual difficulties, difficulty digesting food, sluggish pupil reaction, and exercise intolerance. ${ }^{15}$ Abnormal heart rate variability analysis results have been associated with the early stages of CAN. ${ }^{13}$

In the early 1970s, Ewing and Clarke proposed cardiac autonomic reflex tests based on the heart rate and blood pressure response to physiological maneuvers (eg, Valsalva maneuver, deep breathing, and change in posture) to diagnose CAN. ${ }^{16}$ According to the recommendation of the Cardiovascular Autonomic Neuropathy Subcommittee of the Toronto Consensus Panel on Diabetic Neuropathy, the presence of two or three abnormal results of cardiac autonomic reflex tests (two for borderline, three for definite) was recommended as a criterion for CAN diagnosis based on a scoring system. ${ }^{13}$

The objective of this study performed in diabetic populations was to evaluate a new quantitative sudomotor test for the detection of PDN and CAN, using DNS and CAN scores.

\section{Materials and methods}

In the present study, a medical device managed by software was used: The SudoPath ${ }^{\mathrm{TM}}$ (LD Technology, Miami, FL, USA) (Figure 1). The SudoPath is a programmable electrochemical medical system. The hardware uses two pairs of large stainless steel electrodes connected with cables to the electronic box and placed on the skin where eccrine sweat gland density is very high (the palms of the hands and soles of the feet).

\section{SudoPath background}

Traditional neurophysiologic measurements of sudomotor function include different test modalities, such as quantitative sudomotor axon reflex testing (QSART), thermoregulatory sweat testing, sympathetic skin response, and silastic sweat imprints. The SudoPath is a patented 


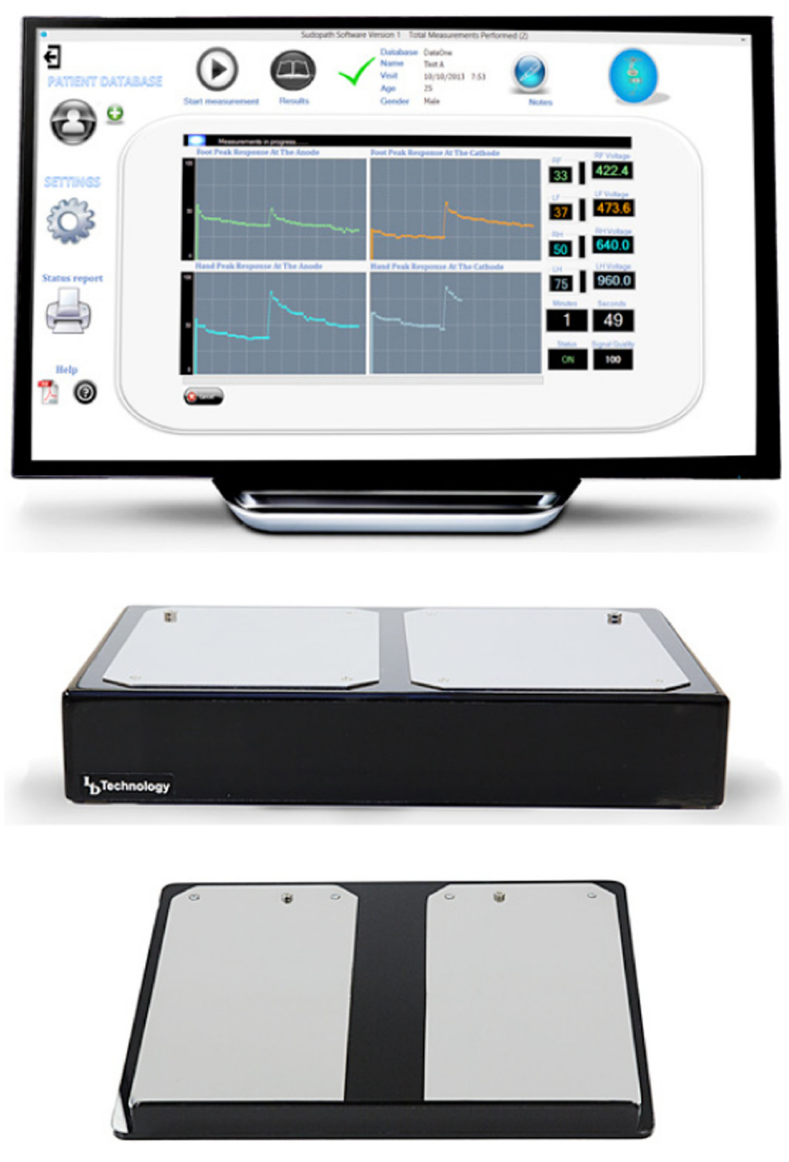

Figure I Photograph of the SudoPath ${ }^{\mathrm{TM}}$

system, and it uses the QSART method, by electrical stimulation of the postsympathetic cholinergic fibers and the switching of polarity, which induces microcirculation vasodilation and the release of chloride ions $\left(\mathrm{Cl}^{-}\right)$via the sweat ducts. The quantitative assessment is performed by measuring changes in conductance caused by electrochemical reactions on the bulk of the stainless steel tactile electrodes.

\section{How does the SudoPath work?}

The electronic box generates a low-voltage output (maximum $1.6 \mathrm{~V}$ ) with a weak direct current (DC) current that is fed to the active electrode and then delivered to the contralateral electrode in two directions for each pathway.

In the general principles of electrolysis, the potential value to reduce $\mathrm{Na}^{+}$ions to sodium is $-2.71 \mathrm{~V}$ and to oxidize $\mathrm{Cl}^{-}$ions to chloride is $-1.36 \mathrm{~V} \cdot{ }^{17}$ The polarity of the contralateral electrode affects which ions are attracted and repelled, which either prevents or allows the output of ions through the sweat gland ducts.

The SudoPath provides three markers of sudomotor function measuring the electrochemical reactions on the bulk of electrodes: 1) peak conductance after the change in polarity (Peak C); 2) conductance at the cathode related to the water reduction; and 3) conductance at the anode related to the water and $\mathrm{Cl}^{-}$oxidation.

\section{Peak of conductance marker}

During the measurement process, the polarity is alternated, which provokes an increase of heat, as well as the delivered voltage to the contralateral electrode (minimum 1.4 and maximum of $1.6 \mathrm{~V}$ ) in the tissue surrounding the sweat glands, and mediate a peak of conductance. Because the heat and increased voltage induce a vasodilation of surrounding vessels, the resulting hyperemia increases skin temperature and thus sets up the sweat response. ${ }^{18}$

\section{Conductance at the cathode marker}

At the cathode, $\mathrm{Cl}^{-}$ions cannot discharge, because they are electronegative. Additionally, due to the low potential generated, $\mathrm{Na}^{+}$ions cannot be reduced at the cathode (as mentioned above, at the time of the switch of polarity the maximum voltage is $1.6 \mathrm{~V})$. Considering this, only the water molecules in the secreted sweat can be reduced. ${ }^{17}$ Therefore, the reduction reaction occurring at the cathode is simply:

$$
2 \mathrm{H} 2 \mathrm{O}+\left(2 \mathrm{e}^{-}\right) \rightarrow(\mathrm{H} 2)+\left(2 \mathrm{OH}^{-}\right)
$$

Because the conductance at the anode is only due to the vasodilation of surrounding vessels, the conductance measured from this phenomenon has been termed the electrochemical skin response - nitric oxide (ESRNO).

\section{Conductance at the anode marker}

The change in polarity during the measurement at the anode increases the voltage, and the $\mathrm{Cl}^{-}$can be oxidized since the voltage is $>1.31 \mathrm{~V}$ and the anode is of opposite polarity. Water molecules and $\mathrm{Cl}^{-}$can be oxidized at the anode. ${ }^{17} \mathrm{The}$ oxidation reaction at the anode is:

$$
\mathrm{H} 2 \mathrm{O}+\mathrm{Cl}^{-} \rightarrow(\mathrm{ClOH})+\left(\mathrm{H}^{+}\right)+\left(\mathrm{e}^{-}\right)
$$

Because the released sweat at the anode contain $\mathrm{Cl}^{-}$ions, we suggest the more probable assumption that the conductance measured at the anode will be related to the release of the $\mathrm{Cl}^{-}$; for this reason, we called the measured conductance at the anode "electrochemical skin response $\mathrm{Cl}^{-}\left(\mathrm{ESRCl}^{-}\right)$" because at a voltage lower than $10 \mathrm{~V}$, the stratum corneum acts as a capacitor. ${ }^{19}$ The only way for the Chloride ions to exit and react with the plate electrode is through the sweat 
ducts. Therefore, $\mathrm{ESRCl}^{-}$could be a valid test of the sweat gland nerve density.

\section{Normal range of the measurement and reproducibility}

The normal range of the SudoPath markers was evaluated with a population supposedly without sudomotor dysfunction. In the tested population, it was found that test fluctuations did not exceed $7 \%$ between two measurements performed at 6-hour intervals.

Regarding the age of the patients, a study by Barontini et al showed a lower skin conductance when comparing young subjects (21 to 40 years) with elderly subjects (61 to 80 years) but not a significant difference of skin conductance when comparing a patient group between the ages of 40 and 80 years. $^{20}$

\section{Measurement protocol}

The subjects were measured in a sitting position to avoid adrenergic sympathetic stimulation that is induced in the standing position. The temperature was maintained at comfortable range (from 73 to 75 degrees Fahrenheit) in order to avoid the activation of sweat glands from the heat or vasoconstriction caused by the cold plates on the extremities.

\section{SudoPath scoring system}

The conductance analysis provides three markers, described in the section "SudoPath background" (Peak C, ESRNO, and $\mathrm{ESRCl}^{-}$), from which a sudomotor response (SMR) score was calculated. Each parameter is scored $0=$ normal, $1=$ borderline, and $2=$ abnormal. A SMR score $>3$ or higher is defined as a positive result for sudomotor dysfunction.

\section{Research plan}

Subjects

A total of 133 type 2 diabetic patients were included in the study (Tables 1 and 2).

\section{Experimental design}

The patients underwent examination at the IPC Heart Care Centre (Mumbai, India) to assess the DNS score, using a questionnaire, and the CAN score, using heart rate variability analysis and blood pressure changes during the Ewing test. In addition, patients were given a sudomotor test using the SudoPath system.

The diagnosis of PDN is based on the DNS score. A DNS score of 1 or higher is defined as a positive result for PDN.
Table I Patient characteristics

\begin{tabular}{|c|c|c|c|}
\hline & $\begin{array}{l}\text { Diabetic with } \\
\text { DNS score } \geq 1 \\
\text { N=35 }\end{array}$ & $\begin{array}{l}\text { Diabetic with } \\
\text { DNS score }=0 \\
\mathbf{N}=98\end{array}$ & $P$-value \\
\hline Men/women & $21 / 14$ & $65 / 33$ & NS \\
\hline Age (years) range & $66(12.1)$ & $56(9.6)$ & $P=0.01$ \\
\hline $\mathrm{BMI}\left(\mathrm{kg}^{2}\right)$ range & $25.8(5.1)$ & $25.3(6.7)$ & NS \\
\hline $\begin{array}{l}\text { Systolic pressure } \\
(\mathrm{mmHg})\end{array}$ & I 43 (24.2) & I4I (I6.4) & NS \\
\hline $\begin{array}{l}\text { Diastolic pressure } \\
(\mathrm{mmHg})\end{array}$ & $82(18)$ & $85(10)$ & NS \\
\hline $\begin{array}{l}\text { Fasting blood glucose } \\
(\mathrm{mg} / \mathrm{dL})\end{array}$ & $128(9.1)$ & $125(8.7)$ & NS \\
\hline
\end{tabular}

Note: Results were expressed as mean (SD).

Abbreviations: BMI, body mass index; DNS, diabetic neuropathy symptoms; NS, not significant; SD, standard deviation.

According to the DNS score, the patients were separated into two groups:

- group 1, comprised of 35 patients (21 males), with the mean age of 66 (range of 56-88) years, who had a DNS score $\geq 1$; and

- group 2, comprised of 98 patients (65 males), with the mean age of 56 (range of 26-85) years, who had a DNS score $=0$.

Patient characteristics are presented in Table 1 and patient statistics in Table 2 . The patients were randomly measured by trained operators throughout the day, without concern of their fasting state.

The inclusion criteria were:

1. age $>18$ years;

2. ability to provide written informed consent; and

3. subject was diagnosed with type 2 diabetes.

The exclusion criteria were:

1. diseases including: hypothyroidism, Raynaud syndrome, Alzheimer disease and Parkinson's disease;

2. treatment with alpha and beta blockers, chemotherapy and corticoids; or

Table 2 Patients' statistics

\begin{tabular}{ll}
\hline & Percent of patients \\
\hline Duration of diabetes & \\
Duration of diabetes $\geq 10$ years & 50 \\
Duration of diabetes $>5$ years and $<10$ years & 35 \\
Duration of diabetes $<5$ years & 15 \\
Treatment of diabetic patients & \\
Metformin & 57 \\
Secretagogue & 63 \\
Insulin & 51 \\
Antihypertensive agents & 65 \\
Antilipidemic agents & 70 \\
Antiplatelet agents & 23 \\
\hline
\end{tabular}


3. any contraindications to the use of the SudoPath systems; use of the system is contraindicated if the patient wears an automatic external defibrillator device or has any implanted electronic device.

The study was conducted according to the ethical principles of the Declaration of Helsinki. All of the subjects provided written informed consent, and confidentiality was maintained for all subjects.

\section{Data analysis}

Statistical analysis was performed using analysis of variance to compare mean differences between the groups, as well as receiver operating characteristic (ROC) curves to determine the specificity and sensitivity of SMR scores to detect PDN, and the coefficient of correlation between the CAN score and SMR score in all the subjects included in the study.

\section{Sample size calculation and statistical analysis}

Based upon the preliminary study results, to have $90 \%$ power to detect a significant difference between subjects with the above mean and standard deviation (SD) at alpha $=0.05,25$ subjects per group needed to be included. The sample size ( $\mathrm{n} \geq 25$ and $\alpha=05 \%$ ) was determined using MedCalc ${ }^{\circledR}$ software (http://www.medcalc.org/publications/ journals.php).

\section{Results}

MedCalc software was used to perform the statistical analysis.

Diabetes patients with a DNS score $\geq 1$ (group 1) had significantly worse mean average scores for the three markers (Peak C, ESRNO, and $\mathrm{ESRCl}^{-}$) for the feet and hands than did the diabetes patient group with a DNS score $=0$ (group 2 ).

Table 3 shows the results of the SudoPath markers and CAN markers comparing the two groups.

When compared with the normal range established in healthy people by SudoPath, more than $76 \%$ of the two groups of diabetic patients had a lower conductance for the three SudoPath markers.

When comparing the diabetes patient group with DNS score $\geq 1$ (group 1) and the diabetes patient group with DNS score $=0$ (group 2), the SMR score had a sensitivity of $91.4 \%$ and specificity of $79.1 \%$ (cutoff $>3$ ) to detect PDN symptoms in diabetic patients $(P=0.0001)$. Area under the ROC curve $($ AUC) $=0.893$ (Figure 2).

A correlation analysis of the total study population returned a coefficient of correlation between the CAN score and SMR score $r=0.68(P<0.0001)$ (Figure 3).
Table 3 Results of mean and standard deviation of SudoPath and CAN parameters

\begin{tabular}{|c|c|c|c|}
\hline $\begin{array}{l}\text { Sudomotor } \\
\text { tests, CARTs, } \\
\text { and HRV analysis }\end{array}$ & $\begin{array}{l}\text { Diabetic with } \\
\text { DNS score } \geq 1 \\
N=35\end{array}$ & $\begin{array}{l}\text { Diabetic with } \\
\text { DNS score }=0 \\
\mathbf{N}=98\end{array}$ & $\begin{array}{l}\text { t-test } \\
\text { significance } \\
\text { level }\end{array}$ \\
\hline Peak C & $61(8)$ & $79(6)$ & 0.0001 \\
\hline ESRNO & $29(4)$ & $42(6)$ & 0.0001 \\
\hline ESRCl & $31(2.4)$ & $45(5)$ & 0.0001 \\
\hline Valsalva ratio & $1.06(0.10)$ & $1.17(0.18)$ & 0.0008 \\
\hline Deep breathing & $1.12(0.05)$ & $1.17(0.04)$ & 0.0001 \\
\hline $\mathrm{K} 30 / 15$ & $1.08(0.04)$ & I.II (0.06) & 0.0069 \\
\hline SPRS & $-1.5(12.5)$ & $-2.01(14)$ & NS \\
\hline HRV total power & $532(63)$ & 745 (72) & 0.0001 \\
\hline LF/HF & $2.1(0.16)$ & $1.64(0.14)$ & $0.000 \mathrm{I}$ \\
\hline
\end{tabular}

Note: Results were expressed as mean (SD).

Abbreviations: CAN, cardiovascular autonomic neuropathy; CART, cardiac autonomic reflex test; DNS, diabetic neuropathy symptoms; $\mathrm{ESRCl}$, electrochemical skin response $\mathrm{Cl}^{-}$; ESRNO, electrochemical skin response - nitric oxide; HF, high frequency of the HRV spectral analysis; HRV, heart rate variability; $\mathrm{K} 30 / / 5$, ratio of heart rate intervals standing at 30 seconds/heart rate intervals standing at 15 seconds; LF, low frequency of the HRV spectral analysis; NS, not significant; Peak C, peak conductance after the change in polarity; SD, standard deviation; SPRS, systolic pressure response to standing.

\section{Adverse events}

No adverse events were reported with the use of the device during the study.

\section{Discussion}

In the present study, $26 \%$ of the diabetic patients were diagnosed with PDN, which is consistent with a cohort study. ${ }^{6}$

The characteristics of the diabetic groups were similar except for age (mean 66 versus 56 years). However, there was no significant difference in skin conductance measurements in this age range, as shown in the Barontini et al study. ${ }^{20}$ Therefore, when comparing similar-characteristic diabetic groups, the SudoPath had good sensitivity and acceptable specificity for detecting the PDN, with AUC $=0.858$. The lower specificity could be explained by the fact that sudomotor dysfunction is the earliest clinically detectable stage of PDN, and therefore the DNS score could be $=0$ at this stage. Also, we found a good correlation between the SMR score and CAN score in all the subjects included in the study.

The results are consistent with the findings of a study using another sudomotor testing device, named Sudoscan, showing that the methods have the ability to detect PDN, with AUC $=0.86$, and also CAN. ${ }^{21}$ The Sudoscan device uses the same sudomotor test method measuring the skin conductance values at the hands and feet. However, the sequence and voltage input are different than for SudoPath and only allow for the assessment of the sweat gland nerve density.

The Sudoscan device showed a sensitivity of $78 \%$ and specificity of $92 \%$ to detect diabetic peripheral neuropathy. ${ }^{21}$ 


\begin{tabular}{|l|l|}
\hline Variable & SMR score \\
\hline Classification variable & Diagnosis \\
\hline Sample size & 133 \\
\hline Positive group & 35 \\
\hline Negative group & 98 \\
\hline Disease prevalence (\%) & $26.3 \%$ \\
\hline AUC & \\
\hline AUC & 0.893 \\
\hline Standard error & 0.0304 \\
\hline 95\% confidence interval & 0.816 to 0.946 \\
\hline Z statistic & 12.936 \\
\hline Significance level $P$ (area $=0.5)$ & $<0.0001$ \\
\hline
\end{tabular}

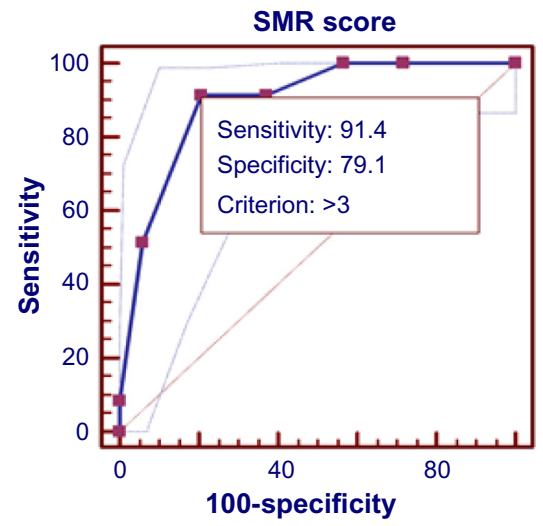

Figure 2 SMR score ROC curve and statistical analysis table comparing diabetic patients with peripheral distal neuropathy symptoms versus diabetic patients without peripheral distal.

Abbreviations: AUC, area under the ROC curve; ROC, receiver operating characteristic; SMR, sudomotor response.

The high specificity of Sudoscan to detect PDN (92\%) could be explained by the following reasons: 1) the control group comprised healthy subjects, 2) the mean age of the two groups was not provided in the study, and 3) the cutoff of the conductance was higher than for SudoPath.

In the present study we compared only diabetic patients and not healthy subjects because, as another study has shown, diabetic patients, even without PDN, have sudomotor dysfunction and lower skin conductance compared with healthy subjects, and could therefore have biased the result of the study. ${ }^{22}$

SudoPath uses a score which includes three components of sudomotor function (sweat output, microcirculation response, and sweat gland nerve density) and has a higher sensitivity than Sudoscan to predict the risk of neuropathy

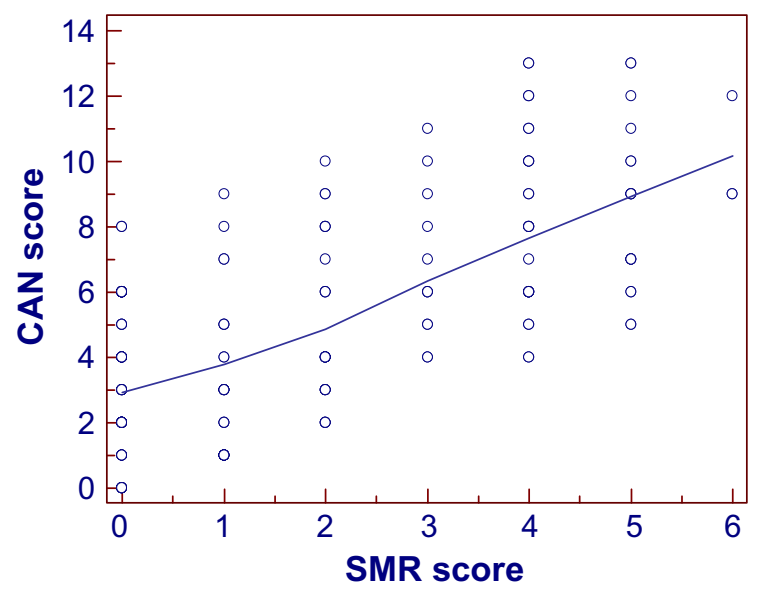

Figure 3 Scatter diagram showing the correlation of CAN score/SMR score. Abbreviations: CAN, cardiovascular autonomic neuropathy; SMR, sudomotor response. in diabetic patients; thus it will be useful as a screening tool before further time-consuming and expensive tests are performed.

The new guidelines from the American Diabetes Association and European Association for the study of Diabetes show the complexity of diabetes treatment and prevention of diabetes complications. ${ }^{23,24}$ The ACCORD study suggests that tight control actually increases the risk for mortality, due to hypoglycemia. ${ }^{25}$ In addition, a recent study on the 10-year follow up of type 2 diabetic patients has shown that CAN increased the risk of hypoglycemia during diabetes treatment. ${ }^{14}$ Therefore, in this context, the SudoPath is very important to consider when formulating and managing treatment regimens.

The SudoPath system is easy to use, operator-independent, and fast (3-minute test time), and it will be useful to assess the susceptibility of patients with type 2 diabetes to develop neuropathy. Furthermore, since glycemic target therapies must be individualized, according to the American and European diabetes associations, the SudoPath could help the physician in diabetes treatment management.

\section{Conclusion}

This study showed that the SudoPath system will be useful to assess the susceptibility of patients with type 2 diabetes to develop neuropathic complications, and also in diabetes treatment management.

These findings should be confirmed by large-scale studies using the SudoPath system and multiparametric analysis to obtain a score for peripheral neuropathy (eg, Toronto Clinical Neuropathy Score). 


\section{Acknowledgment}

The authors want to thank LD Technology for providing the medical devices that were used during the study.

\section{Disclosure}

The authors report no conflicts of interest in this work.

\section{References}

1. Low VA, Sandroni P, Fealey RD, Low PA. Detection of small-fiber neuropathy by sudomotor testing. Muscle Nerve. 2006;34(1):57-61.

2. Kilo S, Berghoff M, Hilz M, Freeman R. Neural and endothelial control of the microcirculation in diabetic peripheral neuropathy. Neurology. 2000;54(6): 1246-1252.

3. Stansberry KB, Hill MA, Shapiro SA, McNitt PM, Bhatt BA, Vinik AI. Impairment of peripheral blood flow responses in diabetes resembles an enhanced aging effect. Diabetes Care. 1997;20(11):1711-1716.

4. Illigens $\mathrm{BM}$, Gibbons $\mathrm{CH}$. Sweat testing to evaluate autonomic function. Clin Auton Res. 2009;19(2):79-87.

5. Alport AR, Sander HW. Clinical approach to peripheral neuropathy: anatomic localization and diagnostic testing. Continuum (Minneap Minn). 2012;18(1):13-38.

6. Abbott CA, Carrington AL, Ashe H, et al; North-West Diabetes Foot Care Study. The North-West Diabetes Foot Care Study: incidence of, and risk factors for, new diabetic foot ulceration in a community-based patient cohort. Diabet Med. 2002;19(5):377-384.

7. Daousi C, MacFarlane IA, Woodward A, Nurmikko TJ, Bundred PE, Benbow SJ. Chronic painful peripheral neuropathy in an urban community: a controlled comparison of people with and without diabetes. Diabet Med. 2004;21(9):976-982.

8. Poncelet AN. Diabetic polyneuropathy. Risk factors, patterns of presentation, diagnosis, and treatment. Geriatrics. 2003;58(6):16-18, 24.

9. Vinik AI, Maser RE, Mitchell BD, Freeman R. Diabetic autonomic neuropathy. Diabetes Care. 2003;26:1553-1579.

10. Tesfaye S, Boulton AJ, Dyck PJ, et al. Diabetic neuropathies: update on definitions, diagnostic criteria, estimation of severity, and treatments. Diabetes Care. 2010;33:2285-2293.

11. Dyck PJ, Kratz KM, Karnes JL, et al. The prevalence by staged severity of various types of diabetic neuropathy, retinopathy, and nephropathy in a population-based cohort: the Rochester Diabetic Neuropathy Study. Neurology. 1993;43(4):817-824.

12. Meijer JW, Smit AJ, Sonderen EV, Groothoff JW, Eisma WH, Links TP. Symptom scoring systems to diagnose distal polyneuropathy in diabetes: the Diabetic Neuropathy Symptom score. Diabet Med. 2002;19(11) 962-965.
13. Spallone V, Ziegler D, Freeman R, et al; Toronto Consensus Panel on Diabetic Neuropathy. Cardiovascular autonomic neuropathy in diabetes: clinical impact, assessment, diagnosis, and management. Diabetes Metab Res Rev. 2011;27(7):639-653.

14. Yun JS, Kim JH, Song KH, et al. Cardiovascular autonomic dysfunction predicts severe hypoglycemia in patients with type 2 diabetes: a 10-year follow-up study. Diabetes Care. 2014;37(1):235-241.

15. Spallone V, Ziegler D, Freeman R, et al. Cardiovascular autonomic neuropathy in diabetes: clinical impact, assessment, diagnosis, and management. Diabetes Metab Res Rev. 2011;27:639-653.

16. Ewing DJ, Clarke BF. Diabetic autonomic neuropathy: present insights and future prospects. Diabetes Care. 1986;9(6):648-665.

17. Grimmes S, Martinsen ØG. Bioimpedance and Bioelectricity Basics. 2nd ed. Oxford: Academic Press; 2008.

18. Sommer P, Kluschina O, Schley M, Namer B, Schmelz M, Rukwied R. Electrically induced quantitative sudomotor axon reflex test in human volunteers. Auton Neurosci. 2011;159(1-2):111-116.

19. Chizmadzhev YA, Indenbom AV, Kuzmin PI, Galichenko SV, Weaver JC, Potts RO. Electrical properties of skin at moderate voltages: contribution of appendageal macropores. Biophys J. 1998;74(2 Pt 1):843-856.

20. Barontini M, Lázzari JO, Levin G, Armando I, Basso SJ. Age-related changes in sympathetic activity: biochemical measurements and target organ responses. Arch Gerontol Geriatr. 1997;25(2):175-186.

21. Casellini CM, Parson HK, Richardson MS, Nevoret ML, Vinik AI. Sudoscan, a noninvasive tool for detecting diabetic small fiber neuropathy and autonomic dysfunction. Diabetes Technol Ther. 2013;15(11): 948-953.

22. Ishibashi F, Kojima R, Kawasaki A, Yamanaka E, Kosaka A, Uetake H. Correlation between sudomotor function, sweat gland duct size and corneal nerve fiber pathology in patients with type 2 diabetes mellitus. J Diabetes Investig. 2014;5(5):588-596.

23. Inzucchi SE, Bergenstal RM, Buse JB, et al. Management of hyperglycemia in type 2 diabetes: A patient-centered approach: position statement of the American Diabetes Association (ADA); European Association for the Study of Diabetes (EASD). Management of hyperglycemia in type 2 diabetes: A patient-centered approach. Diabetes Care. 2012;35(6):1364-1379.

24. Inzucchi SE, Bergenstal RM, Buse JB, et al. Management of hyperglycemia in type 2 diabetes: a patient-centered approach. Position statement of the American Diabetes Association (ADA) and the European Association for the Study of Diabetes (EASD). Diabetologia. 2012;55(6):1577-1596.

25. Seaquist ER, Miller ME, Bonds DE, et al; ACCORD Investigators. The impact of frequent and unrecognized hypoglycemia on mortality in the ACCORD study. Diabetes Care. 2012;35(2):409-414.
Degenerative Neurological and Neuromuscular Disease

\section{Publish your work in this journal}

Degenerative Neurological and Neuromuscular Disease is an international, peer-reviewed, open access journal focusing on research into degenerative neurological and neuromuscular disease, identification of therapeutic targets and the optimal use of preventative and integrated treatment interventions to achieve improved outcomes, enhanced

\section{Dovepress}

survival and quality of life for the patient. The manuscript management system is completely online and includes a very quick and fair peer-review system. Visit http://www.dovepress.com/testimonials.php to read real quotes from published authors. 